of applying a magnetic field in their experiment. But, for many workers in this area, a sine qua non for establishing a new superconductor is the observation of the MeissnerOchsenfeld (MO) effect - when an element or compound enters the superconducting state, magnetic flux that previously permeated the sample is suddenly excluded. Also, in certain superconductors, applying a magnetic field of sufficient magnitude can suppress superconductivity, and this is exactly the effect seen by Shimizu and colleagues. Although they also hold observation of the MO effect to be "indispensable" and were unable to achieve this, their demonstration of this suppression is nevertheless an important pointer.

Another indication of the onset of superconductivity is a drop in electrical resistance, which Shimizu et al. ${ }^{1}$ observed. This, however, is a slightly indeterminate procedure, as can be seen from the authors' data, and future experiments may well lead to some minor revisions of the transition temperature they have measured. Nevertheless, the evidence seems compelling that a superconducting state in lithium has been attained. As the authors note, the reported transition temperature makes lithium (for now) the element with the highest superconducting transition temperature - with, so to speak, not a $d$-electron in sight.

Although it may be regarded as a simple element in atomic terms, in structural terms lithium in its condensed state at high pressures is complex. It seems to be the case $^{5}$ (and indeed it was predicted ${ }^{6}$ ) that, under pressure, lithium takes on structures quite unlike the familiar body-centred-cubic phase, including structures that have an even number of atoms in the unit cell. How this structural complexity connects with the theory of superconductivity is an intriguing question, for perhaps one of the most difficult theoretical problems in the field of condensed-matter physics is accurately predicting superconducting transition temperatures. Nevertheless, general arguments (based on the density of states, the strength of the electron-phonon interaction, the scale of the Coulomb pseudopotential, and so on) can bolster the expectation that a state of significant superconductivity will occur, and did in fact make a strong case for lithium ${ }^{6}$.
But calculations ${ }^{7}$ of lithium's transition temperature seem to come up with a value that is a factor of four or so above Shimizu and colleagues' measurement. In a way, this discrepancy could be seen as a propitious opportunity - the pressure experiments on lithium and on other light-element systems may at last offer the prospect for systematic studies of the behaviour of the dynamic electron-electron interaction and in particular of the Coulomb pseudopotential, the manifestation of direct electron-electron repulsion that generally works against electron pairing (unlike the electron-phonon interaction, which in lithium probably promotes it).

It may be that the early Matthias rule should simply be rephrased to read that single-band, rather than single-valence, elements are in general not favoured for superconductivity, for there is accumulating evidence that structures resulting in multiple bands are predominant among superconductors. If structural anisotropy in layered structures is a route to higher-temperature superconductors, as Ginzburg and Kirzhnits have suggested ${ }^{8}$ and as seems to be the case with the superconducting latecomer $\mathrm{MgB}_{2}$

Behavioural ecology

\title{
Excuses for avian infidelity
}

Over the past decade, molecular methods have provided ample evidence that, in most apparently monogamous animal species, it is quite common for a couple to raise offspring that resulted from either the male or the female mating with another partner. This is particularly evident in birds. Yet explanations for why some female birds actively seek copulations with other males, or why males mate with other females and allow them to lay eggs in the male's nest, are speculative. Elsewhere in this issue, however, a group of ten biologists from six countries provide convincing evidence that, in some cases, the phenomenon can be explained by how closely related the male and female of a pair are (D. Blomqvist et al. Nature 419, 613-615; 2002).

The authors found that in three shorebird species, including the western sandpiper (Calidris mauri), pictured here, the birds of a pair that is rearing 'illegitimate' offspring are more closely related to each other than the average relatedness. So it seems plausible that they sought alternative mates to reduce the harmful effects of inbreeding. Relatives often carry the same versions (alleles) of various genes, so there is a strong chance that their offspring will inherit two identical alleles - one from each parent of those genes. This matters for recessive alleles, which are expressed only if both copies are the same.

Increased expression of recessive alleles depends on the degree of relatedness of the parents and the frequency of the alleles in the population. The effect is greater for rarer alleles - and one would expect alleles with harmful effects to be both rare and recessive. This means that inbreeding is usually associated with increased expression of harmful alleles and a low survival rate of offspring, and therefore that mating with relatives should be avoided.

So there is a logic to Blomqvist and colleagues' finding that shorebirds that are, through some social constraint, paired to a relative then seek alternative partners. The strategy would increase the fitness of offspring and could be evolutionarily selected for. The big question now is, how do the birds know who their mate is? It is likely that many birds can recognize individuals that grew up in the same nest, but we have no idea how they

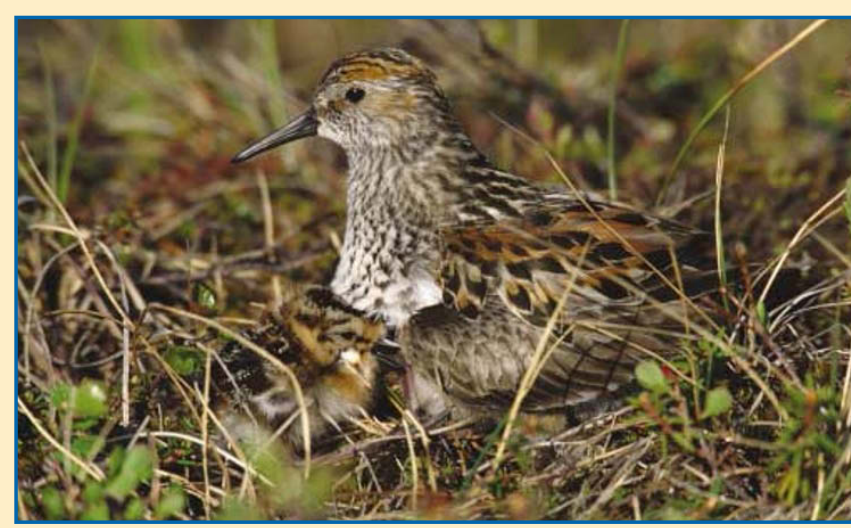

recognize half-siblings from other nests, or cousins. In rodents, smell provides clues, but in most birds the sense of smell is comparatively underdeveloped.

However, Blomqvist et al.'s observation fits with other evidence that birds are cleverer and learn more from past experiences than previously thought. For instance, birds can assess the breeding success of other individuals from the same species, and use that information in deciding where to breed the following year (see, for example, B. Doligez et al. Science 297, 1168-1170; 2002). They can also adjust the time that they reproduce according to past experience (F. Grieco et al. Science 296, 136-138; 2002). All of these findings provide better insight into bird ecology, but also sound a cautionary note to researchers: the idea that birds react to, and compensate for, experimental treatments has become highly plausible. It has long been known that birds are clever and flexible in their behaviour. But we have apparently seriously underestimated them. Arie J. van Noordwijk

Arie J. van Noordwijk is at the Netherlands Institute of Ecology, PO Box 40, NL6666ZG Heteren, The Netherlands.

e-mail:a.vannoordwijk@ nioo.knaw.nl 